\title{
Safety Massage Transmission in VANET using FUZZY based Priority Scheme
}

\author{
Harjit Kaur \\ Research Scholar at \\ department of Computer \\ Science \& Engg. \\ Sachdeva Engg. College for \\ Girls, Gharuan
}

\author{
Preety Chaudhary \\ Assistant Professor at \\ department of Computer \\ Science \& Engg. \\ Sachdeva Engg. College for \\ Girls, Gharuan
}

\author{
Rakesh Kumar \\ Assistant Professor at \\ department of Computer \\ Science \& Engg. \\ Sachdeva Engg. College for \\ Girls, Gharuan
}

\begin{abstract}
VANET is designed for the wireless environment. Nodes are connected and exchange their information via wireless. Therefore some security measure must be considered in communication. Time Critical: The information in VANET must be delivered to the nodes with in time limit so that a decision can be made by the node and perform action accordingly.In this paper the main issue of road density is due to high load on road message communication get overhead due to less amount of network bandwidth to overcome this issue cognitive radio bandwidth can be utilize for data transmission by channel sensing and message can be transmit through cognitive radio channels.
\end{abstract}

\section{Keywords}

GPSR, VANET, simulation models, communication in vehicles, QOS parameters.

\section{INTRODUCTION}

The VANET is the application of MANET. The VANET through the wireless communication network between vehicles takes place. In VANET which vehicles act like as nodes in the network the types of the communications are following. Vehicle to Vehicle (V2V), Vehicle to Infrastructure (V2I) and Vehicle to Roadside (V2R). VANET is the most important component of intelligent transportation system (ITS) in this type of component used to vehicles are prepared with some short range and some medium range wireless communication.

VANET is very important vehicle into a wireless router or node allowing vehicles into connect the turn create a network with wide range network.

The primary goal of vanet is to increase rode safety, improving transportation system and increasing vehicle safety. To accomplish this vehicles act as sensors and replace warnings that enable the drivers to react early to anomalous and potentially dangerous situations like accidents, traffic jams. Instead of safety applications it is also provides comport applications to road users. For example internet access, ecommerce and multimedia applications. Through internet access users can download music, send emails and play games. There are various applications that were developed under collaboration of various government and car manufacturers some of them are "Advance
Desire Assistance Systems (ADASE2), Crash Avoidance Matrices Partnerships (CAMP), CARTACK 2000 and "Fleet Net"

Vehicle to Vehicle (V2V) - in the Vehicle to Vehicle communication is suitable for short range vehicular network. It is provides the real time safety, fast and reliable. It does not need any roadside transportation. It is not very useful in case of sparsely connected network or low density vehicular network. In the $\mathrm{V} 2 \mathrm{~V}$ warning messages are transmit from vehicle to vehicle.

Vehicle to Roadside (V2R) - in the Vehicle to Roadside provides the communication among vehicles and the roadside units. It makes use of pre-existing network infrastructure such as wireless access points. In V2R warning messages are send to roadside units and then from that roadside units warning messages send to the vehicles.

Vehicle to infrastructure - in the vehicle to infrastructure communication provides longer-range vehicular network. It is Provide to easily accusable through the network.

\subsection{GPSR Routing Protocol}

The GPSR is typical routing protocol based on ecological position which is appropriate for VANET network, different from AODV, DSDV, or DSR. GPSR obtains neighbor vehicle node information by GPS positioning equipment, etc. relatively than receiving a large number of routing information in order to maintain the information in neighbor table as well as the information in routing table. The protocol is put forward by Brad Kard and H.T Kung in 2000, the forwarding strategy of which combines greedy forwarding policy with perimeter forwarding policy. In the GPSR routing protocol, each node periodically broadcasts its own location information to the neighbor node the information is stored in neighbor table after neighbor information has been received. The forwarding node chooses the next hop forwarding node according to the both neighbor location information and destination location. Before one node sending data packets, firstly, the id if target node is inquired through location based service information in order to gain the real location of the destination node. Then the data grouping ready for sending is attached with the above obtained geographic information and send to the target node in accordance with greedy forwarding method or perimeter forwarding method. 


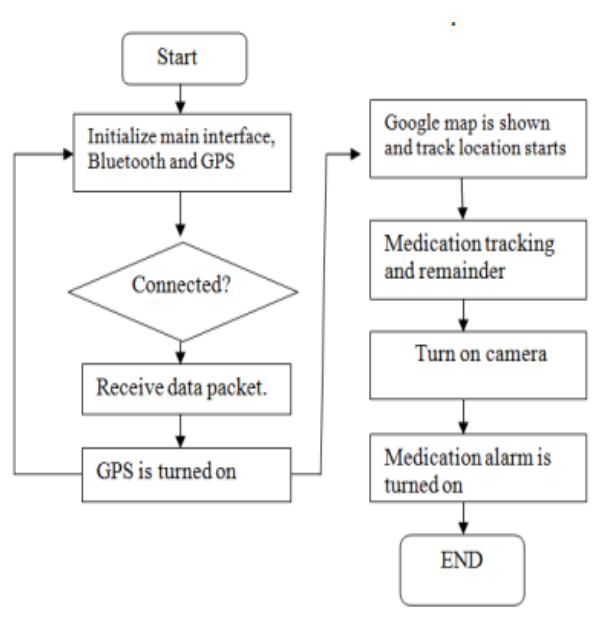

Fig 1.1 flow chart for GPSR routing protocol

\section{RELATED WORK}

In this paper author proposed the combinations one is the GPSR with the grid location service HRGLS hybrid routing and grid location service and another one is GPSR with hierarchical location service called HRHLS hybrid routing or hierarchical location service.

In This paper represent routing packets GPRS used the location information, to find exact destination position the packet is send to the old destination position and from that old position local location request send to get back the exact position.

In this the HLS and GLS algorithms are altered by HRHLS and HRGLS in which old position is used to forward data packet then intermediate node send location request to find the new destination.

In this paper authors discussed proposed the routing technique which is amalgamation of geographic routing protocol greedy perimeter stateless routing and Hierarchical location service. The routing packets are handled by the GPSR protocol or hierarchical location service is used to find the destination position. The problem arises in this is location overhead when the source and destination are far away so the combination of the GPSR protocol and HLS service will reduce the overhead and improve the network performances.

In this paper the authors proposed a hybrid approach which is the combination of the GPSR protocol and the HLS location based service. In movement prediction based joint routing and location based service with the help of predictable position route to the destination is found. To reach the expected destination position cell with the help of in-between nodes it uses the old route but it has a drawback that if middle node have been moved or changing their speed then it cannot take use the old route to reach the predictable cell for broadcasting and whenever packet reaches at the intermediate nodes these nodes has to check the route to the destination causes slow data transfer problem. They also explained the proposed changes in the algorithms in this two algorithms are used firstly Location based service HLS in which two operations are used Poslookup and Predictions Secondly GPSR protocol in which the two operations are GPSRemit and forward packet. The problem can be resolved by choosing the vehicles with relative speed to the source node as intermediate nodes. The comparison of all existing routing protocols is done in table 1 below.

In this paper author deffined Safety is most important in VANET. A major application of VANET is safety counsel. In the VANET Broadcasting of safety messages requires a valuable transmit mechanism. Selection of the search node is the major problem in VANET distribution. The process of the search node selection and broadcasting of safety messages must be achieved in a limited time. for now, the transmission reliability must also be preserved. Multi behavior and Reliable Broadcast (MRB) protocol is especially designed for an best performance of safety applications and addresses these constraints.

\section{PROBLEM FORMULATION}

VANET is an extension of MANET; VANET is vehicular Ad-hoc network which is used for intelligent transport system for the drivers the ad-hoc network is used to transmit various types of message over the network. Safety message has to transmit for the security reasons on the vehicle and road transportation various routing protocols have been utilized for the purpose of message transmission. GPRS, AODV, DSR, PUMA these are various routing protocol utilizes for message transmission vanet scenario is used for mainly V2V and V2R purposes. $\mathrm{V} 2 \mathrm{~V}$ is vehicle to vehicle communications and $\mathrm{V} 2 \mathrm{R}$ is vehicle to roadside communication. In various scenarios message transmission is done according to vehicle density available on the road. Based on the real time road density vehicle establish reliable route for the communication on packet delivery. The main issue of road density is due to high load on road message communication get overhead due to less amount of network bandwidth to overcome this issue cognitive radio bandwidth can be utilize for data transmission by channel sensing and message can be transmit through cognitive radio channels.

\section{METHODOLOGY}

\section{Phase 1}

In this phase VANET scenario is initialize by defining are of simulation no. of vehicle in direction reverse direction their mobility

\section{Phase 2}

In this phase various communications between different vehicles and roadside unit will take place using GPSR protocol for the communication process.

\section{Phase 3}

In this phase cognitive radio bandwidth has been utilized for the transmission of packets from vehicle to vehicle and vehicle to RSU and RSU to vehicle by sensing channel. The channel is free that can be allocated for communication. 


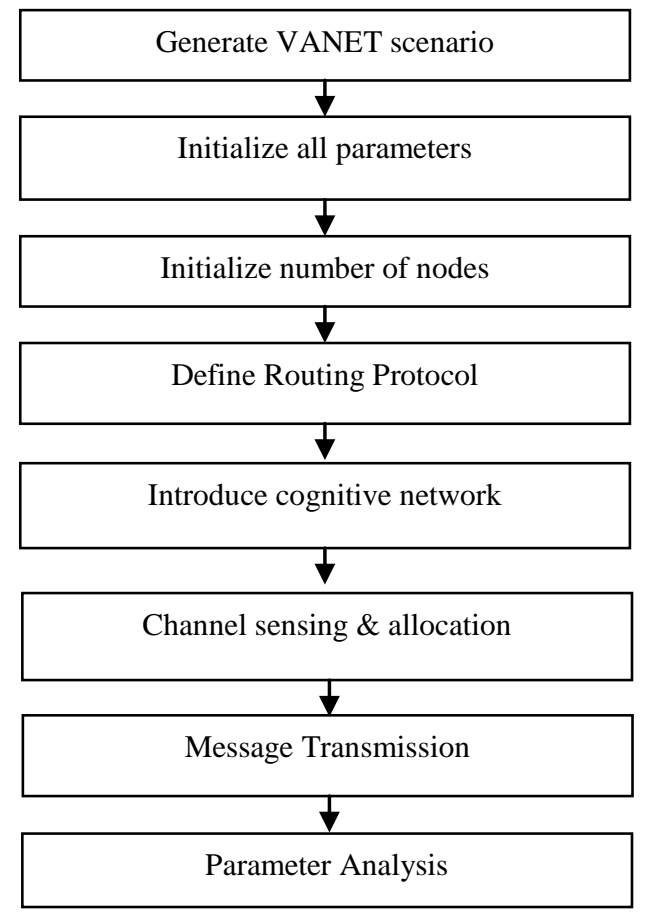

Fig 4.1 Flow chart for purposed work

\section{RESULTS AND DISCUSSIONS}

In the present study, various simulations have performed for getting the results. NS2 simulator runs several times to get the results. In this research work VANET scenario has started by defining traffic simulation number of vehicles in the direction opposite to the direction of their mobility. In running simulation various communications among different vehicles and roadside unit took place using GPSR protocol for the communication process. So, cognitive radio bandwidth is used for the transmission of packets from vehicle to vehicle and vehicle to RSU and RSU to vehicle by sensing channel. The channel is free that can be allocated for communication.

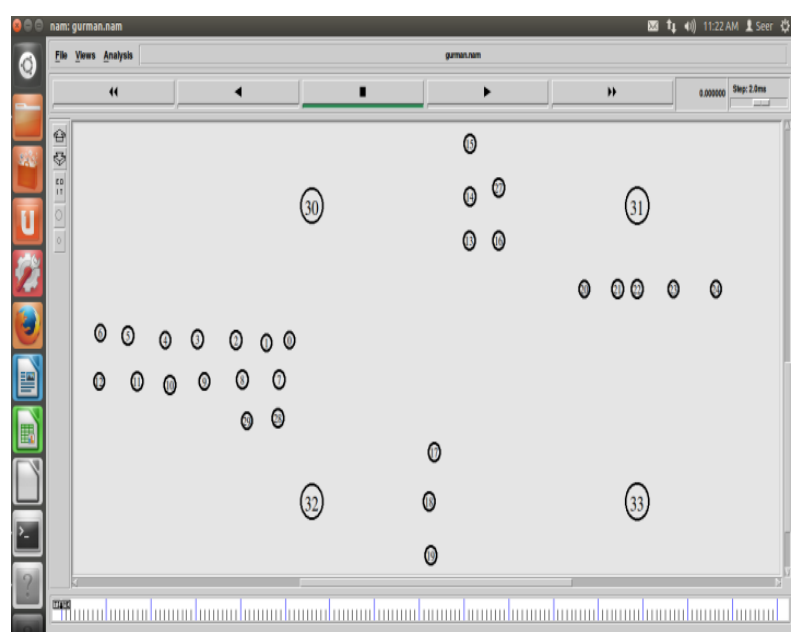

Figure 5.1: Initialization of Nodes

Now in the figure 5.1 nodes are initialized in such a way that they represent VANET scenario. Nodes are in four lanes and four nodes are acting like a road side units (RSU).

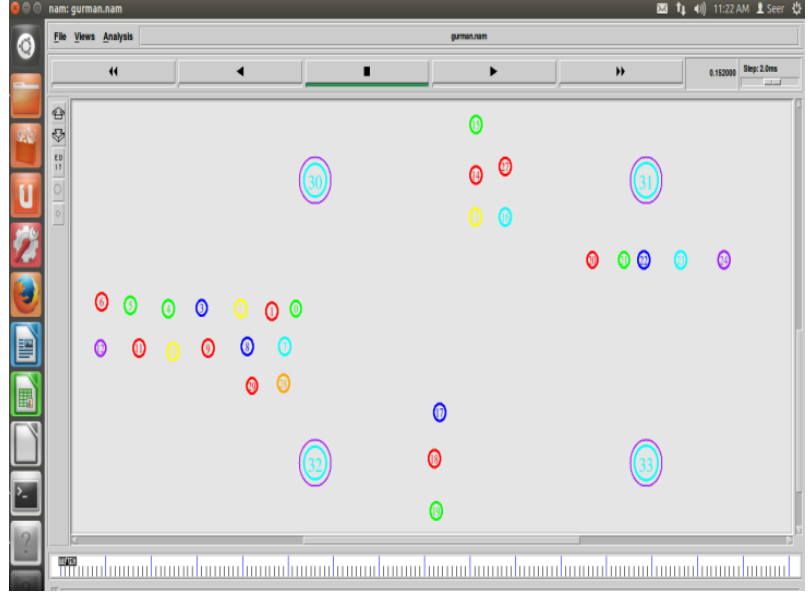

Figure 5.2: Node Representation

The figure 5.2 depicts behavior of nodes as vehicles. They are assumed to be in four different lanes and lane that is moving from left to right is assumed as a high density road. Node number 30, 31, 32, 33 acts as RSUs which communicate with vehicles.

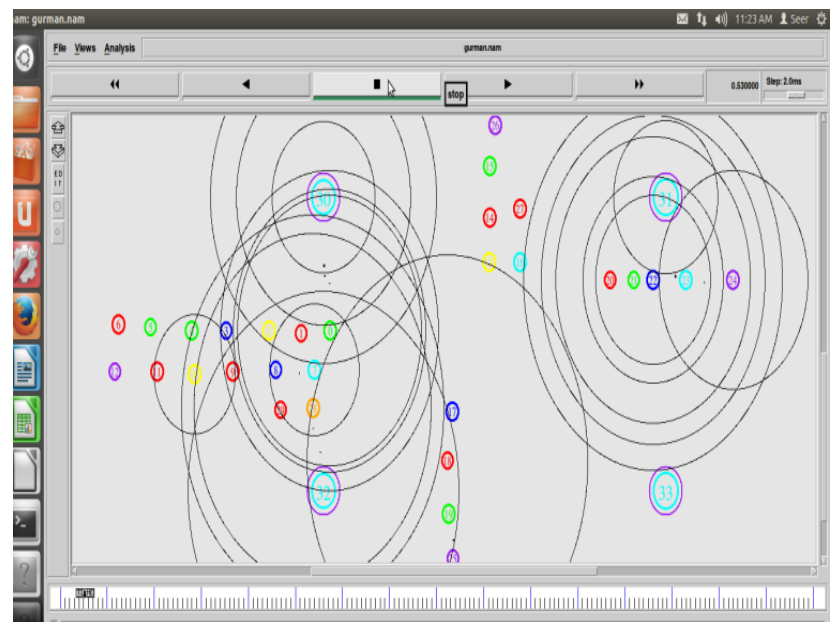

Figure 5.3: Communications between Nodes

In figure 5.3 illustrates scenario that nodes start moving and communicating with each other by passing messages and also communicate with the road side units.

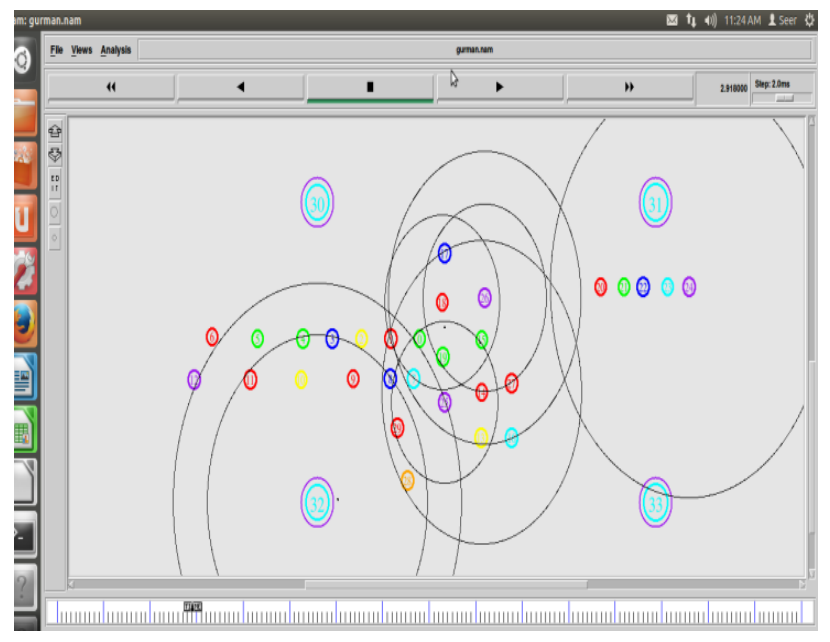

Figure 5.4: Movement of nodes 
Figure 5.4 shows that nodes of upper lane and lower lane crossed because of their green lights and nodes of left lane and right lane have stopped due to their red light.

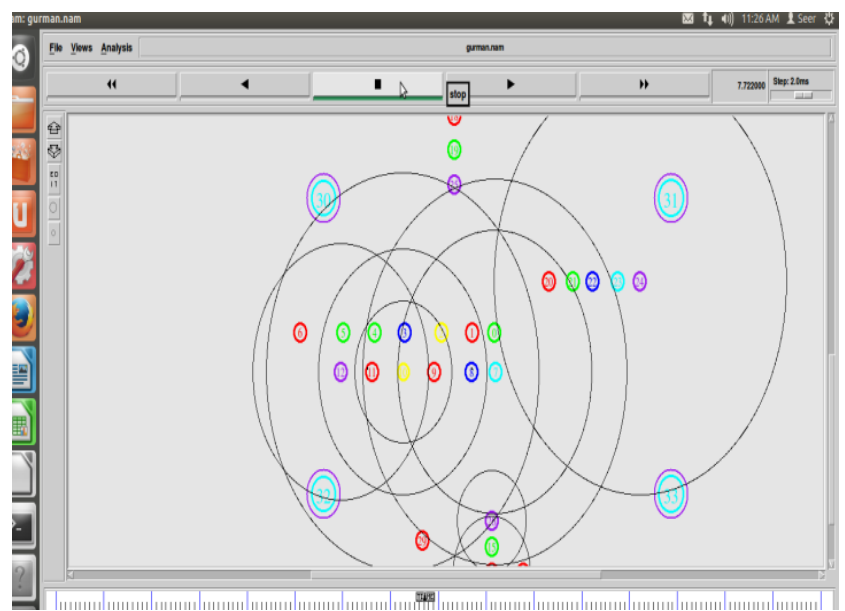

Figure 5.5: Movement of nodes of other lane

Now in the case of figure 5.5, nodes of upper lane and lower lane have crossed and its turn to move nodes of right and left lane and side by they communicate with each other and RSUs.

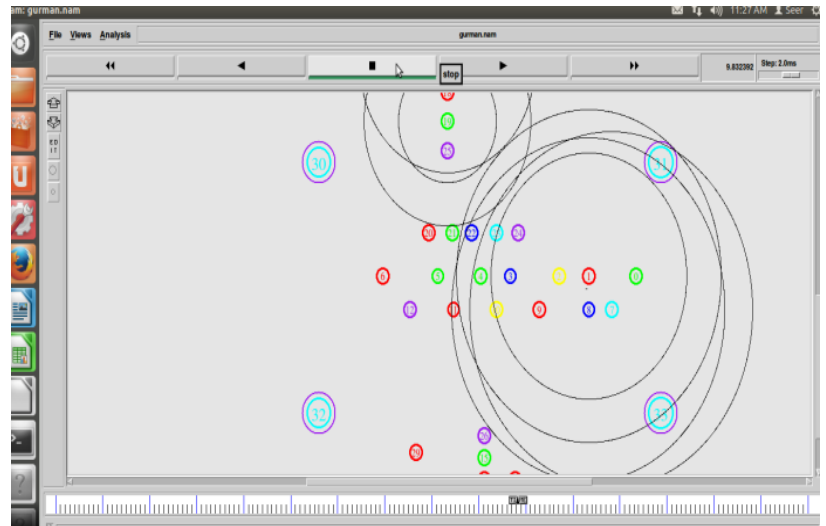

Figure 5.6: Node communications with RSU

The figure 5.6 particularly shows that nodes of left lane which are now moving towards right now start communicate with their new RSU that is node number 31 and 33.

In the graphs, $x$ - axis (horizontal direction) represents the time in seconds and the y- axis (vertical direction) shows that number of bytes transferred in a particular time.

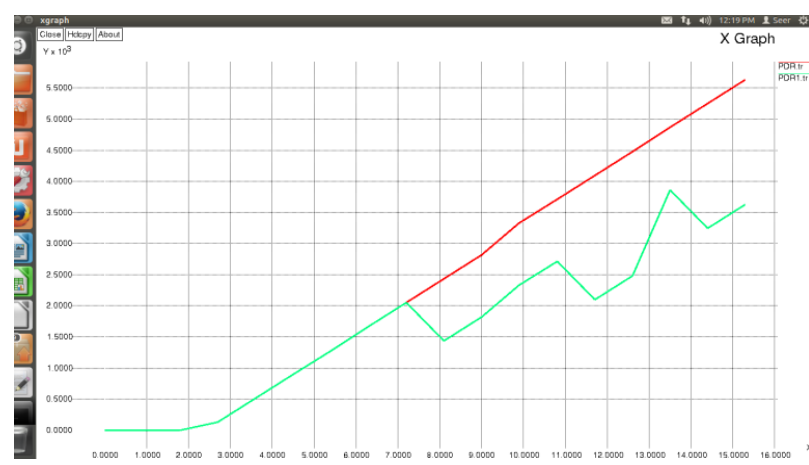

Figure 5.7 Comparison of Packet Delivery Ratio in GPSR and proposed method

It is the ratio of all the received data packets at the destination to the number of data packets sent by all the sources. It is calculated by dividing the number of packet received by destination through the no. of packet originated from the source.

$\mathrm{PDR}=(\mathrm{Pr} / \mathrm{Ps}) * 100$

Graph in figure 5.7 shows the packet delivery ratio for network which shows that firstly when nodes just start communicate their PDR is idle then as they communicate this ratio goes on. On the other hand PDR for existing work is represented by green line which is less as compare to this research.

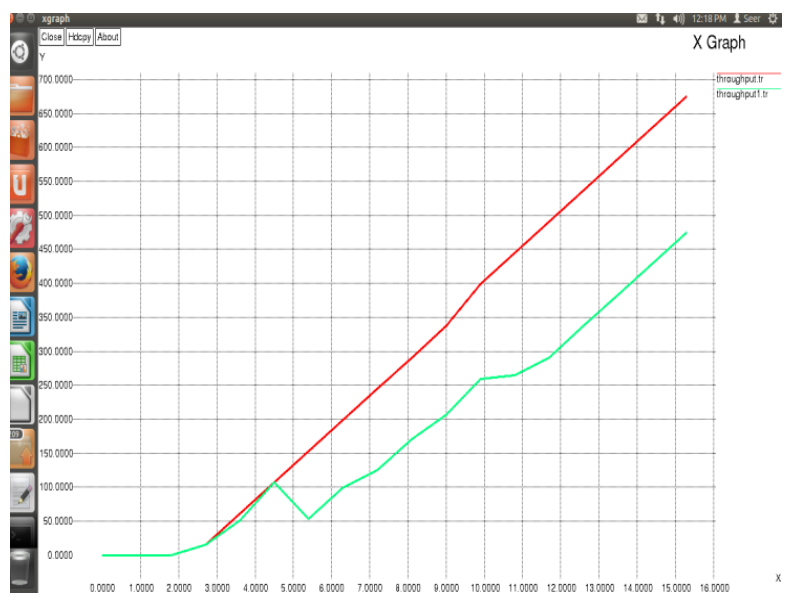

Figure 5.8: Comparison of Throughput for packet delivery in GPSR and proposed method

It is the average at which data packet is delivered successfully from one node to another over a communication network. It is usually measured in bits per second.

Throughput $=($ no of delivered packets $*$ packet size $) /$ total duration of simulation.

Throughput is a total number of successful bytes received per unit time. So, figure 6.8 shows the calculated throughput for the nodes. In this graph red line represents current work and green line is for existing work which is very less as compare to this work.

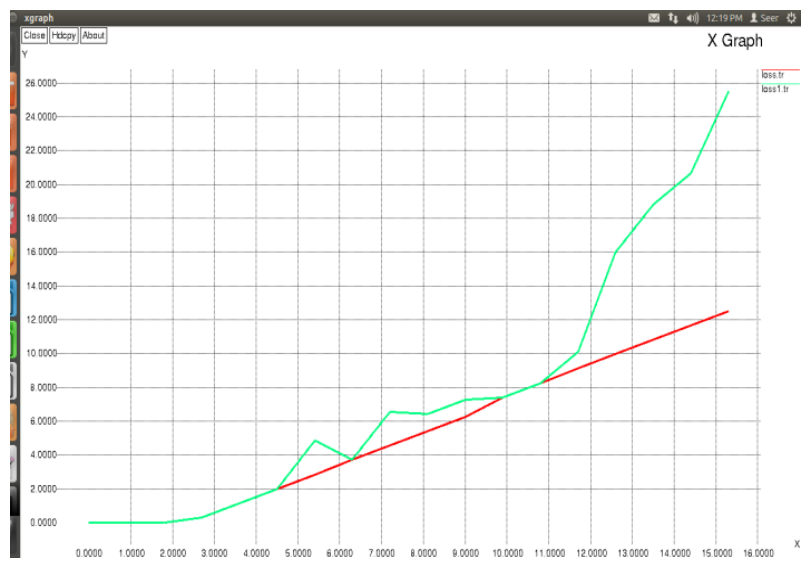

Figure 5.9: Comparison of Packet Loss in GPSR and proposed solution

Packet loss occurs when one or more packets of data travelling across a computer network fail to reach their destination. Packet loss is typically caused by network congestion. Packet loss is measured as a percentage of packets 
lost with respect to packets sent. Loss is defined as the difference between total numbers of bytes sent and total number of bytes received. Figure 6.9 shows that there is very less loss which shows that network is performing well. But on the other hand loss for existing work which is represented by green line is much more as compare to existing one.

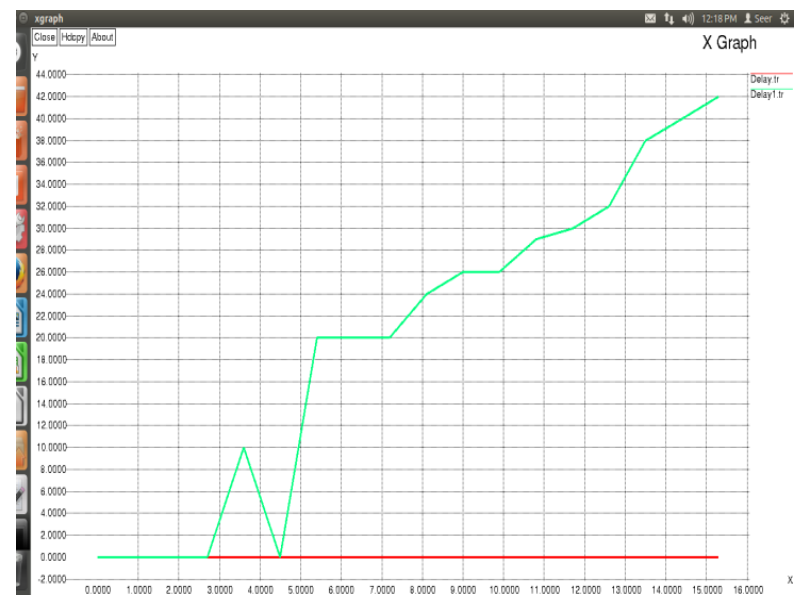

\section{Figure 5.10: Comparison of Delay Time in GPSR and} proposed solution

This includes all possible delays caused by buffering during route discovery, latency, and retransmission by intermediate nodes, processing delay and propagation delay. It is calculated as

$\mathrm{D}=(\mathrm{Tr}-\mathrm{T} \mathrm{s})$

Where, $\mathrm{T} \mathrm{r}$ is receive time and $\mathrm{T} \mathrm{s}$ is sent time of the packet. Delay is the time taken by bytes to reach its destination. Graph in picture shows that delay for this network is very less which means network performance is good but for existing work (represented by green line) delay is very high as compare to current work.

a) Bytes: bytes represents amount of data that has been transmitted from source to destination. These bytes are used for computation of throughput, packet delivery ratio and packet loss of the system.

b) Time: time is the parameter that has been used for evaluation of different values at particular interval of time. Time denotes simulation of the purposed system from staring to end. Time has been measured in micro seconds.

\section{CONCLUSION}

VANET is auto driven system for vehicle that uses various components for automatic transmission of vehicles on road. In VANET roadside unit are available to transmit information over network about vehicle density, position, speed etc.

In the proposed work VANET has been utilized for transmission of safety message over the network by using CRCN. In the proposed work vehicle to vehicle \& roadside unit to vehicle \& roadside unit to roadside unit communication takes place. Cognitive radio utilized channel sensing for availability of vacant channel of radio network. In the proposed work neighbor nodes information has been used for selection of route of message transmission. In the proposed work safety message has been transmitted by using cognitive radio primary channel that assumed message delivery within time.

Various parameters i.e. end to end delay, Packet Delivery Ratio, Throughput has been measured. On the basis of theses parameters one can conclude that Hybrid Approach provides much better results.

\section{REFERENCES}

[1] Alwakeel, S "A virtual P-Persistent bandwidth partitioning manager for VANET's broadcast channel", International conf. on Multimedia Computing and Systems (ICMCS), 2014, PP 1212 - 1215,

[2] Varshney "Security protocol for VANET by using digital certification to provide security with low bandwidth", International Conf. on Communications and Signal Processing (ICCSP), 2014, PP 768 - 772.

[3] Ghosh, T. "Congestion control by dynamic sharing of bandwidth among vehicles in VANET", International Conf. on Intelligent Systems Design and Applications (ISDA), 2014, PP 291 - 296.

[4] Gandhi, U.D "Request Response Detection Algorithm for detecting DoS attack in VANET", International Conf. on Optimization, Reliabilty, and Information Technology (ICROIT), 2014, PP 192 - 194.

[5] Meriam, E. "VANET adaptive and Reliable Broadcast protocol", International Conf. on Wireless Communications and Mobile Computing Conference (IWCMC), 2014, PP 237 - 243.

[6] $\mathrm{Wu}, \mathrm{Sau}-\mathrm{Hsua}$ "A conceptual model and prototype of Cognitive Radio Cloud Networks in TV White Spaces", IEEE Conf. on Wireless Communications and Networking Conference Workshops, 2012, PP 425 - 430.

[7] $\mathrm{Wu}, \mathrm{Sau}-\mathrm{Hsuan}$ "A cloud model and concept prototype for cognitive radio networks", IEEE Conf. on Wireless Communications ISSN 1536-1284, PP 49 - 58, IEEE, 2012.

[8] Wu, Sau Hsuan "A conceptual model and prototype of Cognitive Radio Cloud Networks in TV White Spaces", IEEE Conf on Wireless Communications and Networking Conference Workshops, 2012, pp. 425 430 .

[9] Selvakanmani, S. "CRCN CORMEN - an on demand opportunistic routing protocol for mobile cognitive radio ad hoc networks", IEEE Conf. on Computer Communication and Systems, 2014, pp 265 - 270.

[10] Xing Fu "Cache-Aware Utilization Control for Energy Efficiency in Multi-Core Real-Time Systems", IEEE Conf. on Real-Time Systems (ECRTS), 211, pp. $102-$ 111.

[11] Haiyan Pan "Pulse-width modulation control strategy for high efficiency LLC resonant converter with light load applications", IEEE Conf on Power Electronics, 2014, pp. $2887-2894$.

[12] Haitao Sun "Knowledge for Software Quality Control and Measurement", IEEE conf. on Business Computing and Global Informatization (BCGIN), 2011, pp. $468-$ 470 . 